\title{
COMPARAÇÃO ENTRE MÉTODOS DE ESTABILIZAÇÃO PARA A RESOLUÇÃO DA EQUAÇÃO DE CONVECÇÃO- DIFUSÃO-REAÇÃO
}

\author{
G. F. BARROS ${ }^{1}$, A. M. A. CÔRTES ${ }^{1}$, A. L. G. A. COUTINHO ${ }^{1}$ \\ ${ }^{1}$ Universidade Federal do Rio de Janeiro, Núcleo Avançado de Computação de Alto \\ Desempenho - NACAD/COPPE \\ Email para contato: gabriel.barrosecoc.ufrj.br
}

\begin{abstract}
RESUMO - A equação de convecção-difusão-reação (CDR) está presente em diversos fenômenos físicos, e, na fluidodinâmica computacional, pode ser vista no método $\kappa-$ $\varepsilon$, conhecido em modelagem de turbulência. O presente trabalho compara diversos métodos lineares de estabilização para a resolução da equação de CDR, no intuito de viabilizar o método dos elementos finitos para tal aplicação.
\end{abstract}

\section{INTRODUÇÃO}

Em dinâmica dos fluidos, um regime turbulento é caracterizado por um movimento altamente irregular de um grande número de vórtices que variam em tamanho e interagem entre si (Kuzmin e Hämäläinen, 2015). A modelagem computacional de regimes turbulentos é um assunto debatido de forma intensa nas últimas décadas. Um método já consagrado para modelagem computacional de turbulência, o $\kappa-\varepsilon$ consiste na solução de duas equações de convecçãodifusão-reação (CDR) em conjunto com as equações de Navier-Stokes. O presente trabalho tem como objetivo mostrar como o método dos elementos finitos modela a equação de CDR através do método de Galerkin e como os processos de estabilização lineares podem torná-lo viável.

\section{METODOLOGIA}

A modelagem da equação de convecção-difusão-reação via elementos finitos é delicada, visto que escoamentos predominantemente convectivos (alto $\mathrm{Pe}$ ) e dominantemente reativos (alto $D a$ ) podem apresentar oscilações globais (Joshi e Jaiman, 2017). Uma descrição mais detalhada desses parâmetros é vista na seção 3. O uso do método de Petrov-Galerkin, de forma generalizada, leva à Equação 1, que abre espaço para varios métodos de estabilização. Consequentemente, diferentes operadores de estabilização $\mathcal{L}_{S t}$ (Tabela 1) podem levar a resultados distintos em um mesmo caso.

O operador $\mathcal{L}_{C D R}$ corresponde ao operador linear da equação CDR cujo regime permanente é considerado neste trabalho, visto que a estabilização é relativa à discretização espacial. A modelagem foi implementada em MATLAB. A formulação variacional do problema é: Encontre 
Tabela 1 - Operadores lineares de estabilização

\begin{tabular}{|lll|}
\hline Método & Operador de estabilização $\left(\mathcal{L}_{S t}\right)$ & Trabalho \\
\hline SUPG & $\mathbf{u} \cdot \nabla$ & (Brooks e Hughes, 1982) \\
GLS & $\mathbf{u} \cdot \nabla-\nabla \cdot(\mathbf{k} \nabla)+s$ & (Hughes et al., 1989) \\
SGS & $\mathbf{u} \cdot \nabla+\nabla \cdot(\mathbf{k} \nabla)-s$ & (Hughes, 1995) \\
PPV & $\mathbf{u} \cdot \nabla-\nabla \cdot(\mathbf{k} \nabla)+|s|$ & (Joshi e Jaiman, 2017) \\
\hline
\end{tabular}

$\phi^{h}(\mathbf{x}) \in \mathcal{S}^{h}$ tal que $\forall w^{h} \in \mathcal{V}^{h}$

$$
\begin{aligned}
& \left.\int_{\Omega}\left(w^{h}\left(u \cdot \nabla \phi^{h}\right)+\nabla w^{h} \cdot\left(k \nabla \phi^{h}\right)+w^{h} s \phi^{h}\right)\right) d \Omega+ \\
& +\sum_{e=1}^{n e l} \int_{\Omega} \mathcal{L}_{S t} w^{h} \tau\left(\mathcal{L}_{C D R} \phi^{h}-f\right) d \Omega=\int_{\Omega} w^{h} f d \Omega+\int_{\Gamma N} w^{h} g d \Gamma, \quad \forall w^{h} \in \mathcal{V}^{h}
\end{aligned}
$$

\section{EXEMPLOS NUMÉRICOS}

Alguns exemplos são simulados em uma e duas dimensões. Os números $P e$ e $D a$ representam as relações entre convecção e difusão e reação e difusão, respectivamente. Em uma dimensão, uma malha de 10 elementos é utilizada em um domínio unitário, enquanto em duas dimensões os exemplos são tratados com domínio $\Omega=\left[\begin{array}{ll}0 & 1\end{array}\right] \times\left[\begin{array}{ll}0 & 1\end{array}\right]$ e discretizado em 800 elementos triangulares lineares. Inicialmente, a equação de convecção-difusão é tratada, ou seja, o termo de reação na equação de CDR é nulo. A Figura 1 mostra uma situação com $P e=1$, $f=1$ e condições de contorno de Dirichlet nulas. Observa-se que o valor de $P e$ não é alto o

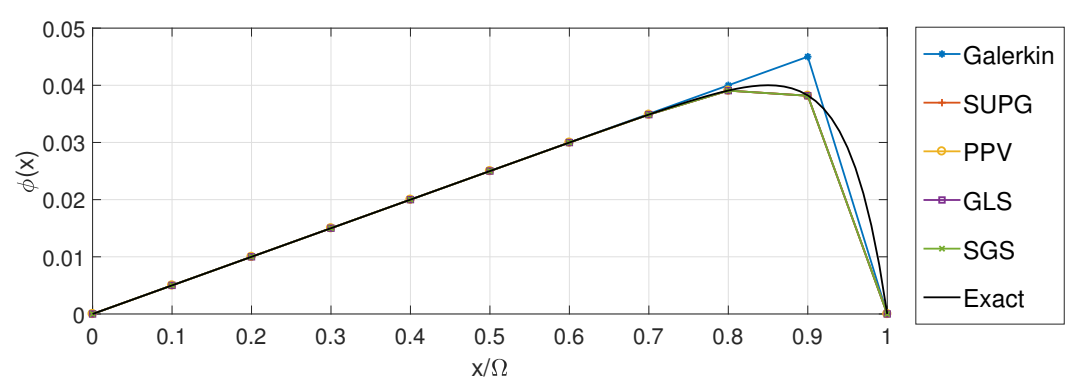

Figura 1 - Equação de convecção-difusão com $P e=1, f=1$ e condições de contorno nulas.

suficiente para instabilizar a solução pelo método de Galerkin, apesar de ser impreciso próximo à condição de contorno da direita. É importante notar também que, nas situações em que o termo de reação é nulo e os elementos são lineares, os operadores dos métodos de estabilização são idênticos, configurando soluções iguais entre todos os métodos estabilizados.

A Figura 2 mostra uma situação com a equação de CDR completa, ou seja, sem termos nulos em sua natureza. O problema corresponde a um transporte com $P e=10, D a=0.1$, $f=0$ e condições de contorno de Dirichlet onde $\phi(x=0)=1$ e $\phi(x=\Omega)=0$. É notável 


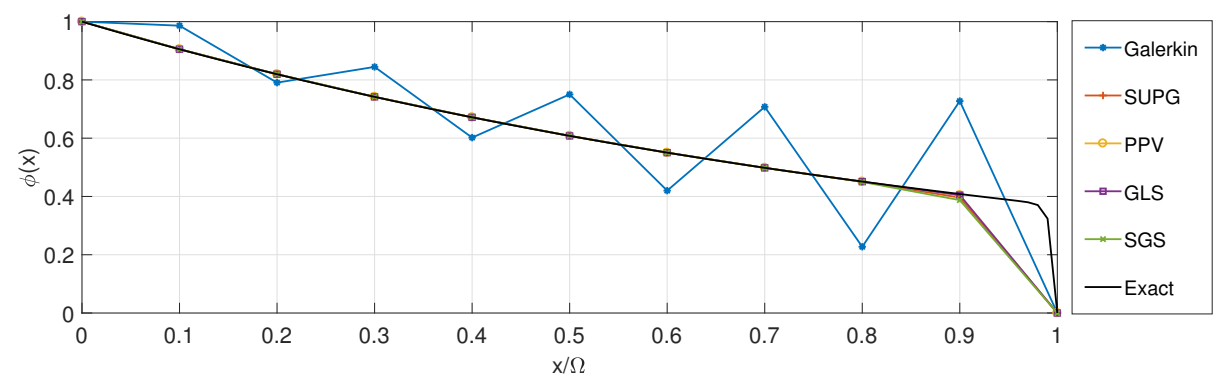

Figura 2 - Equação de convecção-difusão-reação com $P e=10, D a=0.1, f=0$ e condições de contorno de Dirichlet onde $\phi(x=0)=1$ e $\phi(x=\Omega)=0$.

a instabilidade do método de Galerkin para o problema, de forma que nenhum valor nodal obtido com essa configuração se aproximou da solução exata. Os métodos estabilizados, em contrapartida, apresenta solução muito próxima da exata. Há uma pequena divergência entre os métodos, mas a diferença não é relevante para esse problema.

Em duas dimensões, Figura 3 e Figura 4 apresentam a equação de convecção-difusão em que $\mathbf{u}=\left[\begin{array}{l}u_{x} \\ u_{y}\end{array}\right]=\left[\begin{array}{c}10 \\ 0\end{array}\right], f=0, k=0.01$ e as condições de contorno são nulas.

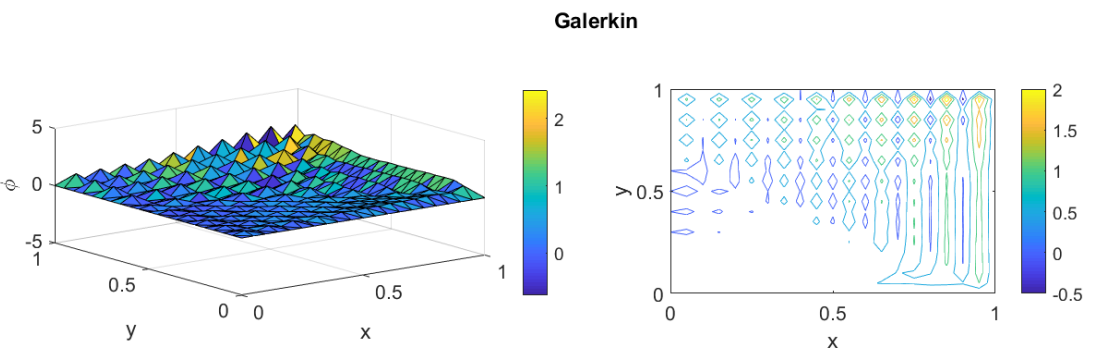

Figura 3 - Solução do problema bidimensional proposto pelo método de Galerkin sem estabilização adicional.

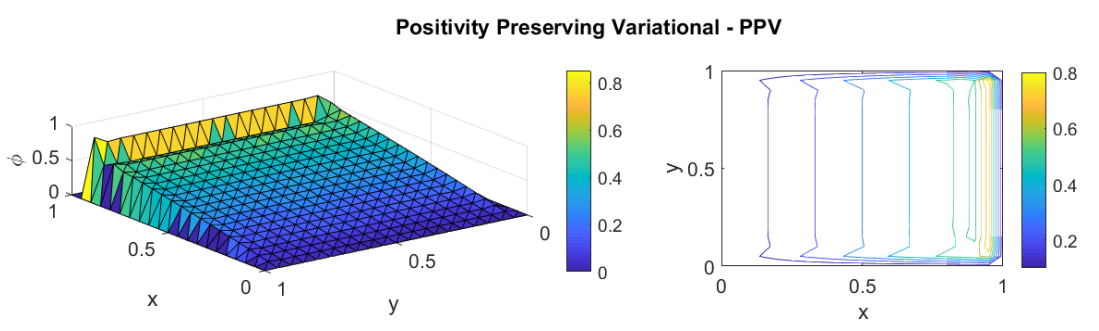

Figura 4 - Solução do problema bidimensional proposto pelo método de Galerkin com estabilização adicional (método PPV).

É possível perceber que a instabilização no método de Galerkin é multidirecional, mesmo 


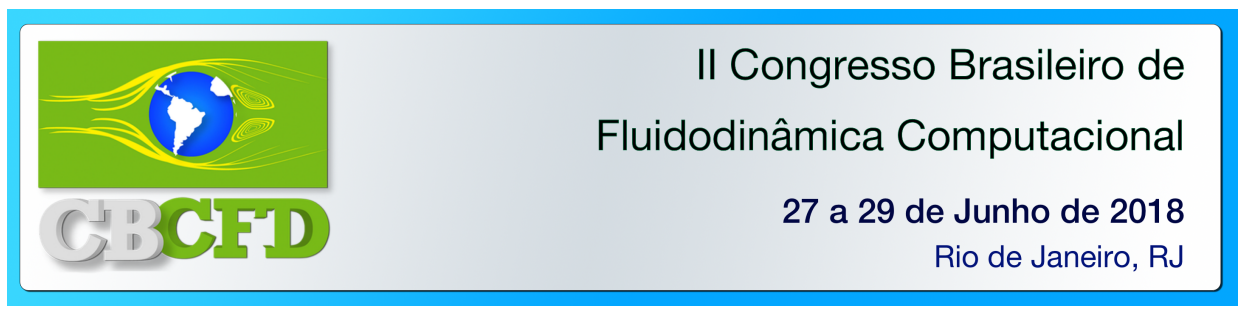

com termos convectivos nulos na direção ortogonal ao fluxo. É interessante observar também o surgimento de uma instabilidade na direção ortogonal ao fluxo no método estabilizado, mesmo que não seja grande o suficiente para prejudicar a modelagem do problema. Isso reflete que a estabilização linear atua somente na direção do transporte convectivo. Uma solução para corrigir esta instabilização se dá na implementação de operadores de captura de descontinuidades não-lineares, garantindo que o acréscimo de difusão seja ponderado no gradiente da solução do problema (Joshi e Jaiman, 2017).

\section{CONCLUSÕES}

Comparando os operadores das estabilizações entre si, é possível perceber que a diferença entre elas nos exemplos citados é pequena se comparadas ao método de Galerkin sem estabilização. O presente estudo mostra a inviabilidade do uso do método de Galerkin para a equação de convecção-difusão-reação, demandando a estabilização do método para garantir que a modelagem seja feita corretamente.

\section{REFERÊNCIAS}

Brooks, A. N.; Hughes, T. J. Streamline upwind/Petrov-Galerkin formulations for convection dominated flows with particular emphasis on the incompressible Navier-Stokes equations. Computer Methods in Applied Mechanics and Engineering, 32(1-3), 199-259, 1982.

Hughes, T. J. Multiscale phenomena: Green's functions, the Dirichlet-to-Neumann formulation, subgrid scale models, bubbles and the origins of stabilized methods. Computer Methods in Applied Mechanics and Engineering, 127(1-4), 387-401, 1995.

Hughes, T. J. R.; FrancA, L. P.; Hulbert, G. M. A new finite element formulation for computational fluid dynamics: VIII. The galerkin/least-squares method for advectivediffusive equations. Computer Methods in Applied Mechanics and Engineering, 73(2), 173189, 1989.

JOSHI, V.; JAIMAN, R. K. A positivity preserving variational method for multi-dimensional convection-diffusion-reaction equation. Journal of Computational Physics, 339, 247-284, 2017.

KuZmin, D.; HÄmÄLÄInen, J. Finite Element Methods for Computational Fluid Dynamics: A Practical Guide, 2015. 